\title{
Maternal cardiac arrest following high neuraxial block
}

\author{
SMK Weerasuriya ${ }^{1 *}$, R Addison ${ }^{2}$, P Sri-Ganeshan ${ }^{3}$ \\ Staff Grade Anaesthetist ${ }^{1}$, Consultant ${ }^{2,3}$, Department of Anaesthesia, Princess Royal University \\ Hospital, Orpington, United Kingdom.
}

*Corresponding author: drsamadara@gmail.com

\begin{abstract}
Maternal cardiac arrest is a dreadful experience for both patient and anaesthetist. This describes a case of maternal cardiac arrest following a high neuraxial block.
\end{abstract}

Keywords: maternal cardiac arrest; high neuraxial block; emergency management; foetal salvage

\section{Introduction}

Maternal cardiac arrest is one of the most dreadful experiences for a patient and anaesthetist. Prompt and vigilant management including peri-mortem caesarean delivery (PMCD) contributes to maternal and foetal wellbeing. We present an obstetric case report of maternal cardiac arrest following high neuraxial blockade.

\section{Case Report}

33 year old primigravida, ASA 2 in established labour had an epidural catheter insertion for labour analgesia. It was straight forward, first attempt procedure with no evidence of dural puncture-confirmed by siphoning test and negative aspiration test. According to Trust protocol, epidural pump was set up for boluses of $15 \mathrm{ml}$ of $0.1 \%$ plain bupivacaine with fentanyl $2 \mathrm{mcg} / \mathrm{ml}$ with lock out interval of 30 minutes. Six hours later she needed to undergo an emergency caesarean section (CS) for foetal distress. It was not specified as category 1 caesarean section initially. But the circumstances gave that impression and later confirmed to be a category 1 CS. The patient was able to transfer herself to the operating table showing absence of considerable motor blockade and she had taken two top ups in the room with effective pain relief. The epidural was topped up in the theatre with $0.5 \%$ levobupivacaine $10 \mathrm{ml}$ and $2 \%$ lignocaine $10 \mathrm{ml}$ (total of $20 \mathrm{ml}$ )over 5 minutes. This was done mainly to avoid a general anaesthetic and to achieve a rapid adequate block.(Two similar caesarean sections were already done in the same way during the same night) Soon afterwards, she complained of difficulty in breathing and showed absence of hand grip and loss of cold sensation to ethyl alcohol below the clavicle. Anaesthetist declared it as a high block, immediately called for help and prepared for intubation. Within seconds, she became cyanosed and emergency management was commenced. Airway was secured, though with difficulty, CPR commenced quickly. As the event was related to local anaesthetic administration, $20 \%$ intralipid $1000 \mathrm{ml}$ was given rapidly. In parallel, obstetric team commenced PMCD immediately and in 2 minutes to the event, delivered a live, non-asphyxiated baby who cried at birth. After further 2 minutes of CPR maternal cardiac output regained. Following that the patient's monitored parameters were stable and no inotropes / vasopressors were required. At the end of the surgery, patient was able to maintain spontaneous breathing. While decisions were made to admit her to the Intensive Care Unit (ICU), she was extubated awake. Amazingly, she was conscious, oriented and was talking appropriately and was pain free. Neuraxial block had descended though still working.The husband, who was distressed, was summoned to the theatre to see her and both were reassured. She spent five hours in the ICU under close monitoring and returned to the Obstetric unit. Later discussions revealed that she remembered only the last few words before losing the cardiac output and there was no awareness. After two days, she presented with severe headache which was successfully treated with epidural blood patch for Post Dural Puncture Headache (PDPH).

\section{Discussion}

Subdural migration of extradural catheter is a recognized complication. In some studies it was confirmed by radiological evidence. ${ }^{1}$ Though this was not done on our patient, the clinical picture, rapidity of block onset and later presentation with PDPH favour the diagnosis. It is possible even 
some local anaesthetic was washed into the intrathecal compartment through unrecognised accidental dural puncture. This case highlights the importance of extreme vigilance in topping up epidurals, recognising problems and early calling for help. Even within the tight time frame of an emergency, checking for existing block and slower topping up while observing for complications become mandatory and should be incorporated in to our practice. Peri-mortem caesarean delivery of the foetus during maternal cardiac arrest enables successful maternal resuscitation and salvage of the foetus. Literature suggests PMCD as the birth of modern day planned caesarean delivery, as before $20^{\text {th }}$ century caesarean delivery was only undertaken if the mother was dead or moribund. ${ }^{2}$ Recommendation to perform caesarean delivery within 4 minutes of maternal cardiac arrest was introduced in 1986 and remains in the guideline for cardiac arrest after 24 weeks of pregnancy with remarkable benefit in maternal resuscitation and salvaging the baby like in this case scenario.

\section{References:}

1. Song J, Shah A, Ramachandran S Rare presentation of accidental subdural block in labour epidural anaesthesia. Open journal of Anaesthesiology. 2012;2;142-145.

DOI: $10.4236 /$ ojanes.2012.24032

2. Roe EJ, Chelmow D. Perimortem Cesarean Delivery. Medscape, Oct 2012. 